\title{
Impact of electronic medical record on physician practice in office settings: a systematic review
}

\author{
Francis Lau ${ }^{1 *}$, Morgan Price ${ }^{2}$, Jeanette Boyd ${ }^{3}$, Colin Partridge ${ }^{4}$, Heidi Bell ${ }^{1}$ and Rebecca Raworth ${ }^{5}$
}

\begin{abstract}
Background: Increased investments are being made for electronic medical records (EMRs) in Canada. There is a need to learn from earlier EMR studies on their impact on physician practice in office settings. To address this need, we conducted a systematic review to examine the impact of EMRs in the physician office, factors that influenced their success, and the lessons learned.

Results: For this review we included publications cited in Medline and CINAHL between 2000 and 2009 on physician office EMRs. Studies were included if they evaluated the impact of EMR on physician practice in office settings. The Clinical Adoption Framework provided a conceptual scheme to make sense of the findings and allow for future comparison/alignment to other Canadian eHealth initiatives.

In the final selection, we included 27 controlled and 16 descriptive studies. We examined six areas: prescribing support, disease management, clinical documentation, work practice, preventive care, and patient-physician interaction. Overall, 22/43 studies (51.2\%) and 50/109 individual measures (45.9\%) showed positive impacts, 18.6\% studies and $18.3 \%$ measures had negative impacts, while the remaining had no effect. Forty-eight distinct factors were identified that influenced EMR success. Several lessons learned were repeated across studies: (a) having robust EMR features that support clinical use; (b) redesigning EMR-supported work practices for optimal fit; (c) demonstrating value for money; (d) having realistic expectations on implementation; and (e) engaging patients in the process.
\end{abstract}

Conclusions: Currently there is limited positive EMR impact in the physician office. To improve EMR success one needs to draw on the lessons from previous studies such as those in this review.

\section{Background}

The need

Increased investments are being made for electronic medical record (EMR) systems to improve physician practice in office settings in Canada. Physician officebased EMR funding support programs are in place in several Canadian provinces [1] and recently there is a pan-Canadian "EMRs and Integration" investment program from Canada Health Infoway [2]. The potential value for EMRs is widely acknowledged, including improved office productivity, care coordination, and patient safety [3]. Yet significant challenges remain in adopting office-based EMRs and reaping the benefits. For instance, two Canadian EMR studies have shown

\footnotetext{
* Correspondence: fylau@uvic.ca

'School of Health Information Science, University of Victoria, P.O. Box 3050

STN CSC, Victoria V8W3P5, Canada

Full list of author information is available at the end of the article
}

that physicians underestimate the need for substantive time commitment, EMR-savvy champions, and ongoing technical/funding support $[4,5]$. Given the current rates of deployment of EMRs in Canada and what is at stake, there is an urgent need to learn from previous EMR studies to determine what had made them successful.

There are systematic reviews on the use of information technology (IT) including EMRs in primary care and general practice settings. These reviews covered topics in diabetes management [6], patient record quality $[7,8]$, decision support tools [9], electronic communication [10], and provider performance and patient outcomes [11]. While there is some evidence of improved quality in such areas as preventive care and guideline adherence, many challenges have been reported [9-11]. These include variable consistency and accuracy of patient record content $[7,8]$, lack of time and funding to cope with change, and the need for 
adequate training and support [6]. While informative, the shortcomings of these reviews are that they were mostly in specific topic areas based on controlled trials published before 2005 (except for [6,9] which were published in 2008). Some of these reviews had a mix of inpatient and outpatient settings, and included large Health Maintenance Organizations with EMRs that are integrated across multiple hospitals and ambulatory care clinics. In contrast, most Canadian physician offices tend to be privately owned solo/group practices or interdisciplinary community-based clinics with standalone EMR systems from small/medium size vendors that are not well integrated with the other health information systems. Different approaches have also been applied to examine the impact of EMRs, including field observation studies [12], workflow analysis [13] and surveys [14]. Thus, there is a need to conduct an EMR review for office settings similar to those in Canada and be more inclusive of different evaluation approaches, covering multiple topic areas $[15,16]$.

This paper describes a systematic review we conducted on EMR-supported physician practice in the office setting. Our questions were: (a) What is the impact of EMRs on physician office? (b) Is there a difference in impact by country, time period and study design? (c) What factors may have led to such impact in the office? (d) What overarching lessons can be drawn to improve EMR success in office-based physician practice? In this review, the Clinical Adoption Framework by Lau et al. [17] was used to make sense of EMR impact and success.

\section{Conceptual model}

The Clinical Adoption (CA) Framework provides a conceptual model to describe the factors that influence health information systems (HIS) success (Figure 1). It extends the Infoway Benefits Evaluation (BE) Framework published in 2006 [18] (adapted from the DeLone and McLean information system success model $[19,20])$. The CA Framework is comprised of micro, meso and macrolevel dimensions. At the micro-level, the CA Framework describes HIS success in terms of HIS quality, use and net benefits. HIS quality includes information, system and service quality; use includes HIS usage and satisfaction; net benefits include care quality, access, and productivity. At the meso-level HIS success is influenced by people, organization and implementation factors. At the macro-level, HIS success is influenced by healthcare standards, legislation/policy/governance, funding/incentive and socio-political and economic trends. The CA Framework was developed with a broad range of HIS in mind, including EMRs.

In this review, we examined EMR impact and success in office settings thru the lens of the CA Framework.
We defined impact as EMR adoption and effect on physician practice, based on evaluation measures used in the studies. For factors that led to such impact we defined them as the observations and/or reasons cited that could explain the adoption and effect. For EMR success we defined it as the improvement that EMRs can make in the physician office. This was similar to reviews that assessed the effects of HIS in other health settings [21,22].

\section{Results}

\section{Synopsis of selected studies}

Our initial search returned 15,042 unique citations. Screening of titles and abstracts left 1,001 articles that required review. While retrieving the full-text for these studies, we removed 66 non-English publications and 170 citations that were not available online (the review team decided not to pursue them due to time constraint). Of the 765 full-text articles screened, 43 studies were selected for this review (27 controlled, 16 descriptive) [12,13,23-63]. See Figure 2.

A synopsis of the 43 studies is shown in Additional file 1 . Twenty-seven of the 43 studies $(62.8 \%)$ were published between 2005 and 2009. Studies from the United States (11 or 25.6\%) and United Kingdom (10 or $23.3 \%$ ) made up half the publications. The remaining studies were from The Netherlands (5 or 11.6\%), Canada (4 or 9.3\%), Australia (4 or 9.3\%), Norway (2 or $4.6 \%$ ), New Zealand (2 or $4.6 \%$ ), and other countries. Fourteen of 43 studies (32.6\%) were focused on work practice, $9(20.9 \%)$ on prescribing support, 7 (16.3\%) on disease management, 6 (13.9\%) on clinical documentation, $4(7.0 \%)$ on preventive care and 4 $(9.3 \%)$ on patient-physician interaction. For study design, 11 (25.6\%) were randomized controlled trial, 7 (16.3\%) quasi-experimental, 9 (20.9\%) observational and $16(37.2 \%)$ qualitative studies. The observational studies included 6 cross-sectional cohort, 1 timemotion, 1 prospective audit and 1 secondary analysis studies. The qualitative studies included 6 multimethod designs, 6 case series, 3 videotape analyses and 1 interview study.

\section{EMR impacts by study and measure}

The 43 studies are summarized by topic area, impacts, influencing factors and our mapping to the CA Framework in Additional file 2. The differences in impact by country, time period and design are reported in Additional file 3. When compared between countries with high versus low adoption rates there was no significant difference found in the ratios of positive studies. There was no difference found between the two time periods of 2000-04 and 2005-09. Odds ratio tests showed more positive results (but not significant) from controlled- 


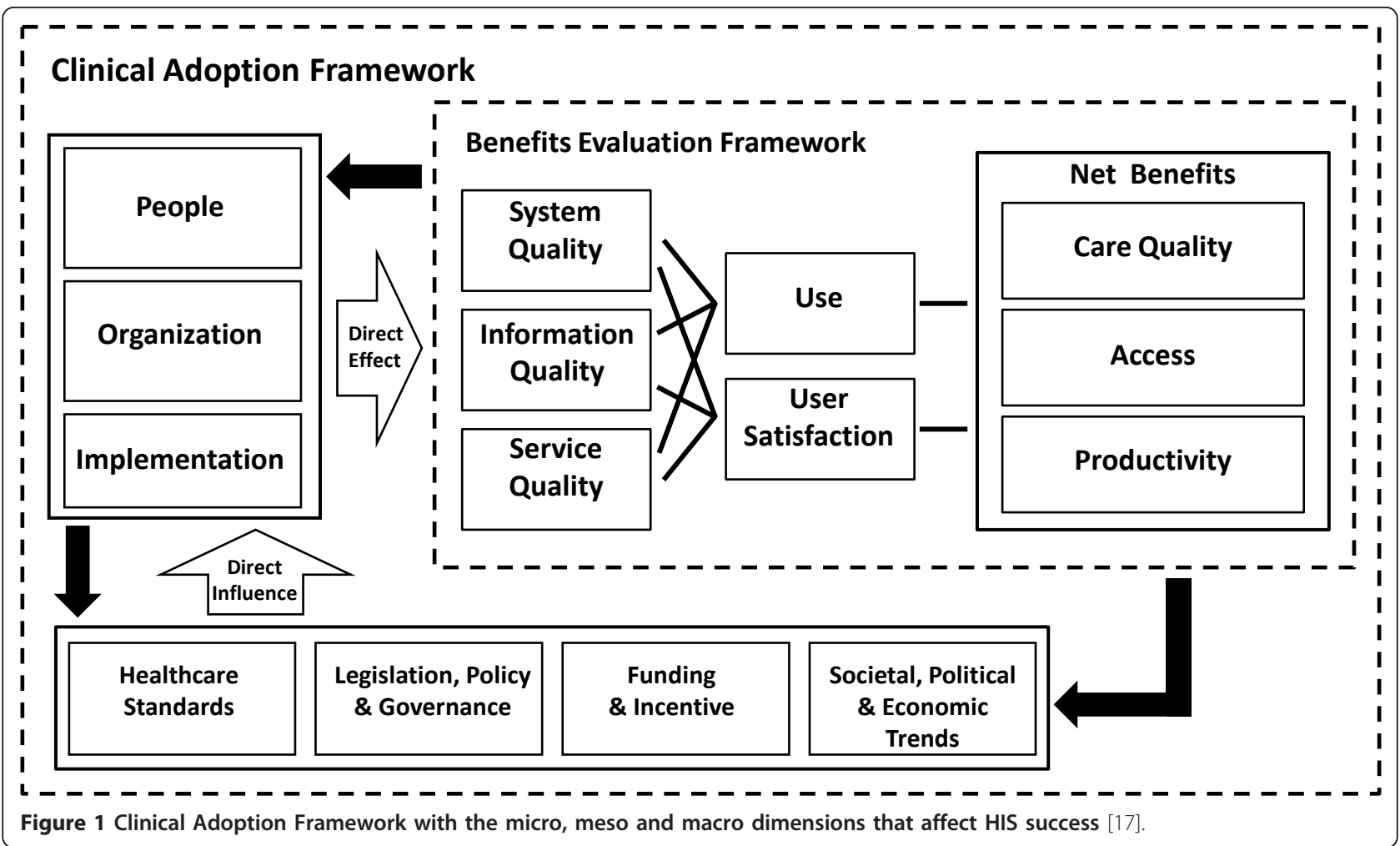

experimental than controlled-observational studies, and no difference between controlled and descriptive studies.

Table 1 summarizes the impacts by study for the six topic areas. For controlled studies, the number of positive counts ranged from highest in work practice $(4 / 5$ studies or $80.0 \%)$ to lowest in clinical documentation (1/ 5 studies or $20.0 \%$ ). For descriptive studies, the positive count was lower in work practice (5/9 studies or $55.6 \%$ ), while the remaining areas had too few study counts for meaningful comparison. When combined, 22/43 studies (51.2\%) had positive impacts, $13 / 43$ studies (30.2\%) had neutral impacts and $8 / 43$ studies $(18.6 \%$ ) had negative impacts. The areas with $>50 \%$ positive counts were preventive care ( $2 / 3$ studies or $66.7 \%)$, work practice $(9 / 14$ studies or $64.3 \%$ ) and disease management (4/7 studies or $57.1 \%)$. The area with the most negative counts was clinical documentation (3/6 studies or $50.0 \%$ ).

The impacts are grouped according to the micro-level dimensions of the CA Framework in Table 2. Odds ratio testing showed only minor differences between the controlled and descriptive studies (i.e., neutral impact, $\mathrm{OR}=2.5 \mathrm{CI} 1.1-5.9$ ). For controlled studies only productivity had $>50 \%$ positive count with 10/16 measures (62.5\%) positive. For descriptive studies the three measures that had $>50 \%$ positive counts were care quality (4/4 measures; $100.0 \%)$, information quality (4/6 measures; $66.7 \%)$ and productivity (11/17 measures; $64.7 \%)$ that were positive. When combined, 50/109 measures
(45.9\%) showed positive impacts, 39/109 measures (35.8\%) showed neutral impacts and 20/109 measures (18.3\%) showed negative impacts. Overall, the only measure that had $>50 \%$ positive count was productivity where $21 / 33$ measures (63.6\%) were positive.

\section{Factors influencing EMR adoption and effect}

A total of 100 factors that influenced EMR adoption and its effect were identified from the 43 studies (see Additional file 2). After merging those that were similar we ended up with 48 distinct factors. These factors were mapped to the categories of the CA Framework [refer to $[17,18]]: 23$ of them were micro-level, 16 meso, and 9 macro (see Figure 3 ). The types of influence are elaborated below.

At the micro-level, system quality factors included the availability of templates $[25,42]$, interface design $[31,36,41,44,48,50,55,57,59]$ and technical performance (e.g. speed and reliability) $[44,55,58]$. Information quality factors included the organization, accuracy, completeness and accessibility of the patient record [23,28,29,35-37,44,45,47-56]. Service quality factors included training and technical support $[53,62,63]$, system backup and unexpected downtime $[52,55]$. EMR usage factors included its intent (e.g. quality improvement versus record keeping) [41], actual strategies for optimal/appropriate use [12-26], ease of use [35,49], and usage patterns that emerged over time [40]. 


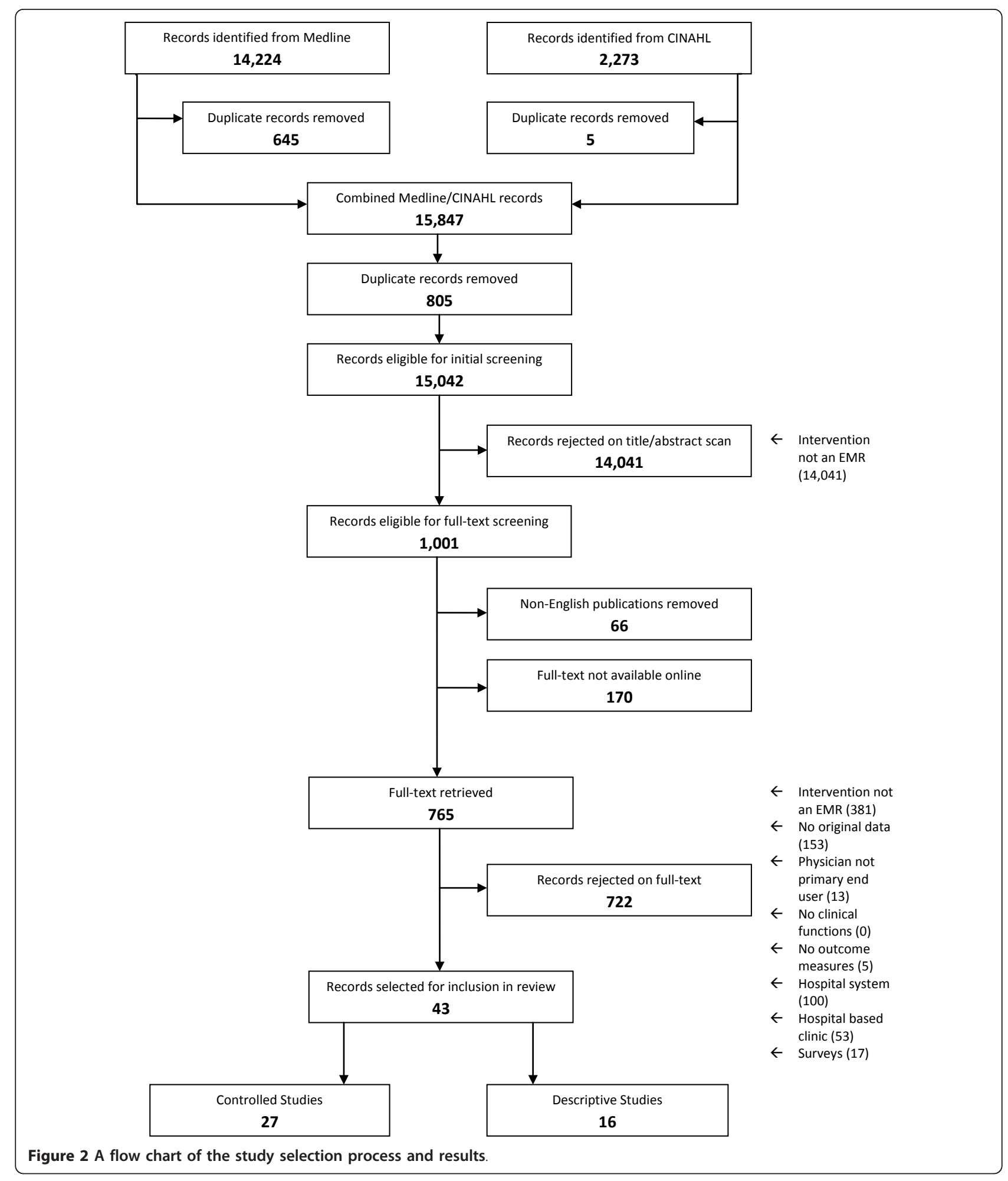

Interaction related factors covered patient-physician encounters such as the type of consult (e.g. psychological) $[12,26,42,43]$, consult room layout [42] and patients' ability to schedule appointments $[27,33]$. For net benefits, care quality factors covered patient safety [38], care effectiveness [40], quality improvement [45] and guideline compliance $[32,57,60,61]$. Productivity factors covered care efficiency $[13,27,38,42,56]$, 
Table 1 Number of positive, neutral and negative impacts by study for the six areas

\begin{tabular}{|c|c|c|c|c|}
\hline Topic Areas & $\begin{array}{c}\text { Positive } \\
(\%)\end{array}$ & $\begin{array}{c}\text { Neutral } \\
(\%)\end{array}$ & $\begin{array}{c}\text { Negative } \\
(\%)\end{array}$ & Total \\
\hline \multicolumn{5}{|l|}{ Controlled } \\
\hline Prescribing Support & $3(37.5)$ & $4(50.0)$ & $1(12.5)$ & 8 \\
\hline Disease Management & $4(57.1)$ & $2(28.6)$ & $1(100.0)$ & 7 \\
\hline Clinical Documentation & $1(20.0)$ & $2(40.0)$ & $2(40.0)$ & 5 \\
\hline Work Practice & $4(80.0)$ & $1(20.0)$ & $0(0.0)$ & 5 \\
\hline Preventive Care & $1(33.3)$ & $1(50.0)$ & $0(0.0)$ & 2 \\
\hline $\begin{array}{l}\text { Patient-Physician } \\
\text { Interaction }\end{array}$ & $0(0.0)$ & $0(0.0)$ & $0(0.0)$ & 0 \\
\hline Sub-total & $13(48.1)$ & $\begin{array}{c}10 \\
(37.0)\end{array}$ & $4(14.8)$ & 27 \\
\hline \multicolumn{5}{|l|}{ Descriptive } \\
\hline Prescribing Support & $1(100.0)$ & $0(0.0)$ & $0(0.0)$ & 1 \\
\hline Disease Management & $0(0.0)$ & $0(0.0)$ & $0(0.0)$ & 0 \\
\hline Clinical Documentation & $0(0.0)$ & $0(0.0)$ & $1(100.0)$ & 1 \\
\hline Work Practice & $5(55.6)$ & $1(11.1)$ & $3(33.3)$ & 9 \\
\hline Preventive Care & $1(100.0)$ & $0(0.0)$ & $0(0.0)$ & 1 \\
\hline $\begin{array}{l}\text { Patient-Physician } \\
\text { Interaction }\end{array}$ & $2(50.0)$ & $2(50.0)$ & $0(0.0)$ & 4 \\
\hline Sub-total & $9(56.3)$ & $3(18.8)$ & $4(25.0)$ & 16 \\
\hline \multicolumn{5}{|l|}{ Combined } \\
\hline Prescribing Support & $4(44.4)$ & $4(44.4)$ & $1(11.1)$ & 9 \\
\hline Disease Management & $4(57.1)$ & $2(28.6)$ & $1(14.3)$ & 7 \\
\hline Clinical Documentation & $1(16.7)$ & $2(33.3)$ & $3(50.0)$ & 6 \\
\hline Work Practice & $9(64.3)$ & $2(14.3)$ & $3(21.4)$ & 14 \\
\hline Preventive Care & $2(66.7)$ & $1(33.3)$ & $0(0.0)$ & 3 \\
\hline $\begin{array}{l}\text { Patient-Physician } \\
\text { Interaction }\end{array}$ & $2(50.0)$ & $2(50.0)$ & $0(0.0)$ & 4 \\
\hline Total* & $22(51.2)$ & $\begin{array}{c}13 \\
(30.2)\end{array}$ & $8(18.6)$ & 43 \\
\hline
\end{tabular}

*Odds ratios between controlled and descriptive studies are not significant

coordination $[24,58]$ and net cost including billing, staffing and maintenance costs $[25,45,46,52]$.

At the meso-level, people factors included personal characteristics and expectations such as prior EMR experience of the users $[34,60]$, and their personal time investment in exchange for the benefits expected from the system $[13,28,42]$. Roles/responsibilities covered the need for champions and staff participation [24,45], and shift in tasks (e.g. documentation by staff vs. physicians) $[13,28]$ that could lead to role ambiguity and conflict [30]. Organization factors included structure/processes and culture that supported EMR adoption/use [23,30,45,51], EMR-practice fit (e.g. hybrid EMR/paper systems) [50], and EMR-supported office and workflow design $[30,45,51,53,56,59,63]$ such as the placement of computer screens in consult rooms [42]. Return-onvalue focused on demonstrated value at the practice level such as substitution effect from guideline driven test orders and prescribing [51], and tangible cost-efficiency gain with larger practice size and patient volume
Table 2 Number of positive, neutral and negative impacts by measure in the CA Framework

\begin{tabular}{|c|c|c|c|c|}
\hline $\begin{array}{l}\text { Topic Areas } \\
\text { Controlled }\end{array}$ & $\begin{array}{c}\text { Positive } \\
\text { (\%) }\end{array}$ & $\begin{array}{c}\text { Neutral } \\
(\%)\end{array}$ & $\begin{array}{c}\text { Negative } \\
(\%)\end{array}$ & Total \\
\hline \multicolumn{5}{|l|}{ Info System Quality } \\
\hline System & $1(33.3)$ & $0(0.0)$ & $2(66.7)$ & 3 \\
\hline Information & $4(33.3)$ & $6(50.0)$ & $2(16.7)$ & 12 \\
\hline Service & $0(0.0)$ & $0(0.0)$ & $0(0.0)$ & 0 \\
\hline \multicolumn{5}{|l|}{ Use/Satisfaction } \\
\hline User Satisfaction & $0(0.0)$ & $0(0.0)$ & $0(0.0)$ & 0 \\
\hline \multicolumn{5}{|l|}{ Net Benefits } \\
\hline Care Quality & $12(37.5)$ & $16(50.0)$ & $4(12.5)$ & 32 \\
\hline Access & & & & 0 \\
\hline Productivity & $10(62.5)$ & $6(37.5)$ & $0(0.0)$ & 16 \\
\hline Subtotal & $27(42.9)$ & $28(44.4)$ & $8(12.7)$ & 63 \\
\hline \multicolumn{5}{|l|}{ Descriptive } \\
\hline \multicolumn{5}{|l|}{ Info System Quality } \\
\hline System & $2(33.3)$ & $0(0.0)$ & $4(66.7)$ & 6 \\
\hline Information & $4(66.7)$ & $1(16.7)$ & $1(16.7)$ & 6 \\
\hline Service & $0(0.0)$ & $0(0.0)$ & $2(100.0)$ & 2 \\
\hline \multicolumn{5}{|l|}{ Use/Satisfaction } \\
\hline User Satisfaction & $2(18.2)$ & $9(81.8)$ & $0(0.0)$ & 11 \\
\hline \multicolumn{5}{|l|}{ Net Benefits } \\
\hline Care Quality & $4(100.0)$ & $0(0.0)$ & $0(0.0)$ & 4 \\
\hline Access & $0(0.0)$ & $0(0.0)$ & $0(0.0)$ & 0 \\
\hline Productivity & $11(64.7)$ & $1(5.9)$ & $5(29.3)$ & 17 \\
\hline Subtotal & $23(50.0)$ & $11(23.9)$ & $12(26.1)$ & 46 \\
\hline \multicolumn{5}{|l|}{ Combined } \\
\hline \multicolumn{5}{|l|}{ Info System Quality } \\
\hline System & $3(33.3)$ & $0(0.0)$ & $6(66.7)$ & 9 \\
\hline Information & $8(44.4)$ & $7(38.9)$ & $3(16.7)$ & 18 \\
\hline Service & $0(0.0)$ & $0(0.0)$ & $2(100.0)$ & 2 \\
\hline \multicolumn{5}{|l|}{ Use/Satisfaction } \\
\hline User Satisfaction & $2(18.2)$ & $9(81.8)$ & $0(0.0)$ & 11 \\
\hline \multicolumn{5}{|l|}{ Net Benefits } \\
\hline Care Quality & $16(44.4)$ & $16(44.4)$ & $4(11.1)$ & 36 \\
\hline Access & $0(0.0)$ & $0(0.0)$ & $0(0.0)$ & 0 \\
\hline Productivity & $21(63.6)$ & $7(21.2)$ & $5(15.2)$ & 33 \\
\hline Total & $50(45.9)$ & $39(35.8)^{*}$ & $20(18.3)$ & 109 \\
\hline
\end{tabular}

*Significant odds ratio $(\mathrm{OR})=2.5, \mathrm{Cl} 1.10-5.90$

[48]. Implementation factors included the extent that the introduction of an EMR into the practice was planned and carried out as a priority project with dedicated time and resources $[52,55,62,63]$. The service support provided during implementation was critical $[48,53,62,63]$, since they affected the disruptions that physicians and office staff had to overcome while learning to use the EMR and redesign their work routines.

At the macro-level, factors under healthcare standards included standardized data content [23,56], established practice guidelines $[32,60]$, and legal documentation requirements $[55,56,59]$ that affected EMR design/ 


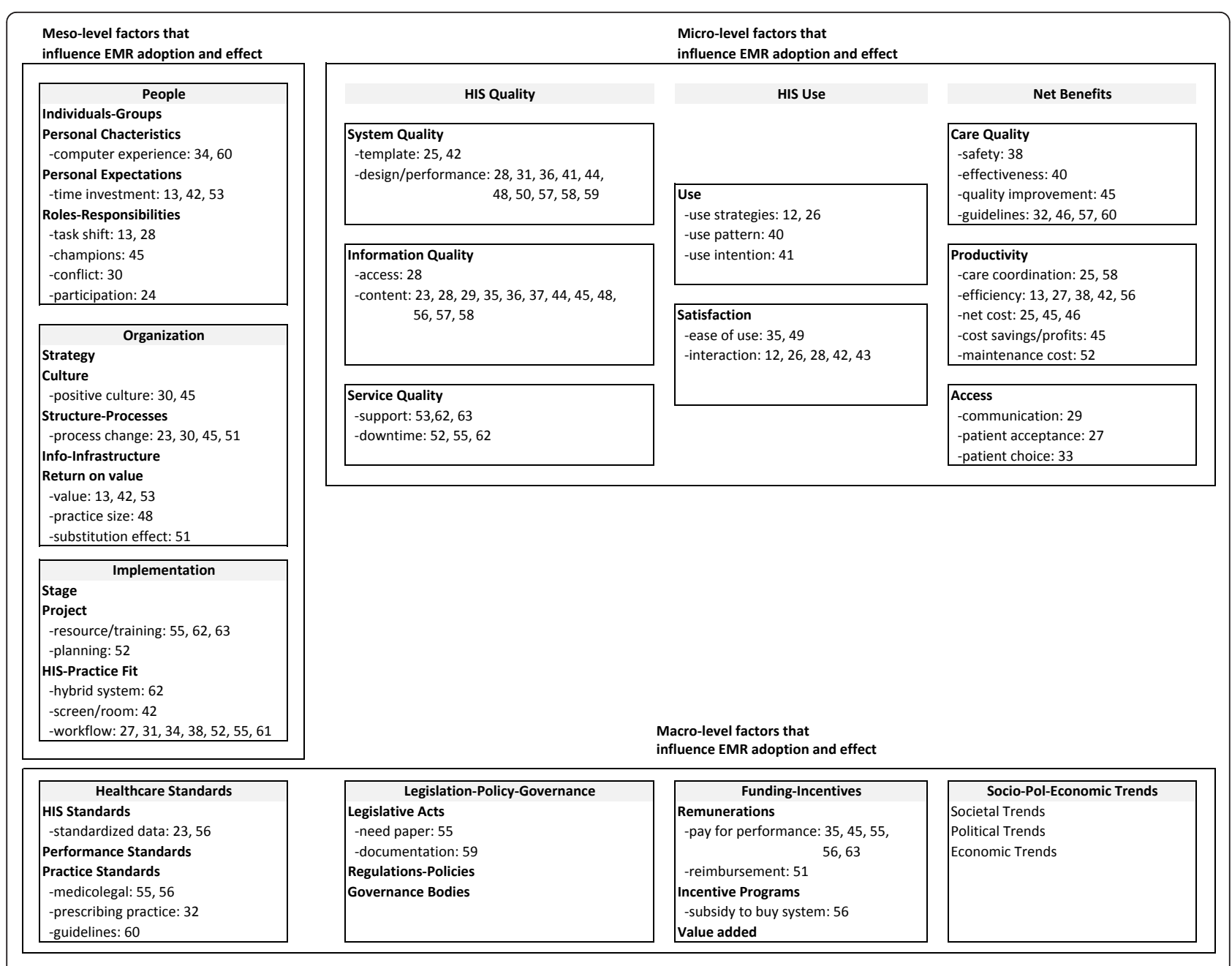

Figure 3 Micro, meso and macro-level factors that influenced EMR success.

performance and user behaviours. They also covered practice standards for clinical guidelines [60], professional scope of practice [32] and medico-legal requirements $[55,56]$ that governed EMR use. Factors under funding/incentives included remuneration schemes such as pay for performance and fee-for-service that encouraged EMR use $[35,45,55,56,63]$ and incentive programs in the form of subsidies to purchase/adopt EMR systems [56].

\section{Summary of key findings}

Overall, this review found 22/43 EMR studies (51.2\%) and 50/109 measures (45.9\%) had shown positive impact across the six topic areas examined. When grouped by area, there were modest improvements in preventive care $(66.7 \%)$, work practice $(64.3 \%)$ and disease management $(57.1 \%)$. Clinical documentation showed the least improvement with EMR use (16.7\%). Within the dimensions of the CA Framework, EMRs had shown a modest improvement in productivity (63.6\%), whereas user satisfaction had the least improvement (18.2\%). About one-third of the studies and measures were not able to show an impact. Less than one-fifth of the studies and measures had a negative impact. No significant differences were found based on adoption rates by country, by time period and by study design.

Through this review we found that EMR impact was influenced by many factors. In particular, we were able to extend the CA Framework to EMRs in physician office settings by identifying specific micro, meso and macro level factors that influence EMR adoption and its effect. For instance, at the micro-level, the EMR's technical design, performance and support affected its usage and user satisfaction in the office. At the meso-level, the implementation process and resulting workflow impacted the office's ability to improve productivity and coordination. At the macro-level, incentives such as pay-for-performance were seen as an important driver for EMR adoption since they increased the return on investment made. 


\section{Discussion}

\section{Making sense of EMR success}

Our review findings suggest there is a $51 \%$ chance that an EMR can improve office practice, while in $30 \%$ of the time there may not be any effect, but only $19 \%$ may lead to negative consequences. Surprisingly, no significant differences in impact were found from studies across countries, time periods and study designs. When compared with the earlier reviews for primary care and general practice settings, our findings showed less improvement in the areas of disease management [6], patient record quality $[7,8]$ and decision support tools [9], but similar improvement in preventive care [11]. When compared with the more general HIS review by Lau et al. [15], which showed reported positive impacts in $62.7 \%$ of studies and $54.4 \%$ of measures, our counts looking at EMRs were lower at $51.2 \%$ and $45.9 \%$, respectively. There was similar improvement in preventive care impacts $(66.7 \%$ vs. $72.1 \%)$ [15], while our review showed greater improvement in work practice (64.3\%).

A further comparison was made with an earlier review by Eslami et al. [64] on inpatient computerized provider order entry (CPOE) systems identified in the Lau review [15]. The inpatient CPOEs had greater positive impacts in guideline adherence, organizational efficiency and user satisfaction than those reported in our EMR review. These findings suggest that there are differences for physicians in office settings that need to be considered; perhaps they face greater challenges with less advanced EMR features and fewer resources available for improvement. While these comparisons place our review results into larger HIS contexts, caution is needed when interpreting these findings since the range of studies, methods, systems and settings examined were highly variable across these reviews.

\section{Extending the CA framework to EMR in office settings}

This review had extended the CA Framework by identifying specific factors that influenced EMR success in the office setting. These factors are also consistent with those reported in the earlier reviews. Examples are the need for adequate time, funding and training [6], accurate clinical documentation $[7,8]$ and computer-supported interactions among physicians and patients $[10,11]$. Other factors are the importance of having computer-prompted alerts/reminders and involving users in system design [21]. When compared with the success factors reported in van der Meijden's review [65] of inpatient clinical information systems, we were able to map many of their factors to those listed under the micro-level dimensions of the CA Framework in our EMR review. In addition, we were able to map all of their contingent factors to those in the meso and macro levels of the CA Framework in our review. These findings suggest that, the adoption and ongoing use of office-based EMRs face many of the same issues as other HIS deployment efforts, although the ways of addressing these issues may be different. In this regard, the CA Framework should be expanded by incorporating post-adoption usage behaviours by physicians and their effects over time to reflect the different maturity stages of the EMRs involved [66,67].

\section{Lessons to guide future efforts}

Based on this review we argue there is much room for improvement. Specifically, we can achieve EMR success by drawing on the lessons from earlier studies examined in this review. The key lessons are: (1) Having robust EMR features that support ongoing clinical use - by paying close attention to interface design (e.g. templates, decision support) and technical performance (e.g. reliability and speed) issues to ensure the efficient and accurate capture/retrie$\mathrm{val} / \mathrm{use}$ of patient data; (2) Redesigning EMR-supported work practices for optimal fit - by reorganizing the clinical workflow to make full use of the advanced EMR features such as patient recalls and electronic referrals; (3) Demonstrating value for money to encourage EMR adoption/use by leveraging incentives such as pay-for-performance in chronic disease management and improving patient safety thru such alerts as drug level monitoring; (4) Having realistic expectations on the EMR implementation effort - by setting tangible goals and putting in the time, resource and commitment needed to achieve them; (5) Engaging patients in the process to improve the overall encounter experience - by involving patients in using the EMR as a communication, information and decision tool before, during and after their office visit.

\section{Study limitations}

There are limitations with this review. First, only online English articles in scientific journals were included; we could have missed studies in other languages, grey literature or hardcopies only. Second, the initial literature search was done by only one team member, which could have introduced bias in the initial screening step. Third, our conceptual model and vote-counting methods used to describe and correlate EMR use, measures and impacts were simplistic, which might not have captured the complexities involved with EMR adoption and their evaluation. In particular the vote counts of articles did not take into account the type of the study design or sample size. Fourth, caution is needed in generalizing our review findings due to the small number of studies selected. Last, our review covered a wide range of complex EMR issues, which might not have been adequately explored and fully explained. 


\section{Conclusions}

Currently there is limited positive EMR impact in physician office practice. The CA Framework, impacts, factors, findings and lessons described in this review provide the necessary components for us to make sense of EMR success in the office practice setting. This review contributes to the overall evidence base on EMR adoption/use to guide future effort.

\section{Methods}

\section{Selection of studies}

We had three practising physicians (MP, JB, CP) on our review team to assist in study selection and synthesis to ensure the relevance of our findings. One researcher (HB) searched two online databases - Ovid MEDLINE ${ }^{\circledR}$ and CINAHL $^{\circledR}$ in early 2010 using search strategies prepared with the assistance of a medical librarian (RW). The search covered combinations of concepts for electronic medical record, office practice, physician and impact. We limited our search to English articles published in the last decade as they were more likely to be relevant than those from earlier periods (from 2000 to 2009). Studies in English were included if they: evaluated use of an EMR in an office-based setting; were based on original data; had physicians as primary end-users; focused on clinical functions; and reported impact on practice performance, patient outcomes, or physician-patient interactions. Studies were excluded if their EMRs were part of the hospital information systems or were a hospital ambulatory clinic settings, or if they were only survey studies. These criteria excluded Health Maintenance Organizations with integrated electronic patient records, such as the United States Veterans Health Administration. After removing duplicates from the combined MEDLINE ${ }^{\circledR}$ and CINAHL ${ }^{\circledR}$ searches one reviewer did the preliminary screening of all citations. Full-text review of the articles was done by two teams of two reviewers (one researcher and one physician per team). The third physician (MP) was the tie-breaker. The final article selection for analysis was done by consensus (FL, HB, MP). Corresponding authors of original articles were contacted by HB to verify the setting if needed (See Additional file 4: Detailed Search Protocol online supplement).

\section{Data extraction/synthesis}

We grouped the selected articles into controlled and descriptive studies according to their design. Controlled studies were further grouped by type of design as experimental or observational. For each study HB extracted data on the author, country, year, study design, participants, intervention, measures and results, which were then confirmed by FL. We organized the studies into six topic areas: prescribing support, disease management, clinical documentation, work practice, preventive care, and patient-physician interaction. These topic areas have been used in the meta-synthesis by Lau et al. [15]. A study could have multiple measures and each measure could independently have a positive, neutral or negative impact. A positive impact is when an EMR was associated with an improvement in the measured effect; a neutral impact is when there was no difference; a negative impact is when the EMR had a worse effect.

To aggregate EMR impacts across studies, we used the "vote-counting" method applied in other HIS reviews $[15,21,22]$ to tally the number of positive studies and impacts present. For controlled studies, a positive impact occurred when the measure showed a significant statistical difference between groups. For descriptive studies, we relied on the authors to report if the measure being examined had a positive impact. In studies with multiple measures, Garg's method [21] was adopted where $\geq 50 \%$ of the impacts should be positive for a study to be counted as positive. The impacts were categorized according to the dimensions of the CA Framework. They were tabulated separately for controlled and descriptive studies and then combined. Odds ratios were used to test for differences in the odds of positive impacts reported between controlled and descriptive studies under each topic before they were combined. The studies were also compared by country (based on adoption rates $>90 \%$ for Europe and Asia Pacific, and $<50 \%$ for North America reported by Schoen et al. [Exhibit-1 in ref [3]]), study design (experimental/observational/descriptive) and time period (2000-04 vs. 2005-09). Related impacts and factors were grouped and reported as key lessons that could influence EMR success. Two reviewers (FL, HB) worked independently on the aggregate analysis and reconciled the outputs through consensus afterwards. One physician (MP) on the review team provided critique to refine the synthesized findings.

\section{Endnotes}

In this review, physicians include family physicians, general practitioners and specialists. Office settings include private offices where physicians work in solo/group practice, and interdisciplinary primary/specialty care clinics

\section{Additional material}

Additional file 1: Appendix A. Summary of Original Studies by Topic.

Additional file 2: Appendix B. Summary of Impacts and Factors.

Additional file 3: Appendix C. Comparison of Studies by Country,

Design and Period.

Additional file 4: Detailed search protocol as online supplement. 


\section{Acknowledgements}

We wish to thank Agnes Wong and Jes Bassi for reviewing earlier versions of this paper. Funding support was provided by the Canadian Institutes for Health Research and Canada Health Infoway, thru the eHealth Applied Chair Award, the College of Pharmacists of British Columbia, and the Department of Family Practice at the University of British Columbia. Note that the review findings are the opinions of the authors alone and do not represent the views of any organization.

\section{Author details}

${ }^{1}$ School of Health Information Science, University of Victoria, P.O. Box 3050 STN CSC, Victoria V8W3P5, Canada. ${ }^{2}$ Faculty of Medicine, University of British Columbia, 5950 University Blvd, Vancouver V6T1Z3, Canada. ${ }^{3}$ Admirals Medical Clinic, 275 Island Hwy, Victoria V9B1G4, Canada. ${ }^{4}$ Kootenay Boundary and Creston Community of Practice, 518 Lake Street, Nelson V1L4C6, Canada. ${ }^{5}$ University of Victoria Libraries, University of Victoria, P.O. Box 1800 STN CSC, Victoria V8W3H5, Canada.

\section{Authors' contributions}

FL was main author for the manuscript and took part in the article screening and synthesis. MP assisted in writing the manuscript and served as tie-breaker in the review process. JB and CP both served as reviewers of the articles. HB conducted the article search, screening, selection and data extraction. RR assisted in the search protocol creation/revisions. All authors read and approved the final manuscript.

\section{Competing interests}

The authors declare that they have no competing interests.

Received: 10 August 2011 Accepted: 24 February 2012

Published: 24 February 2012

\section{References}

1. Canadian Medical Association: Information Technology and Health care in Canada: 2010 Status Report [http://www.cma.ca/Thandbook].

2. Canada Health Infoway: Unlocking the Value of Health Information Systems Corporate Business Plan 2010-2011 [https://www.infoway-inforoute.ca/flash/ lang-en/bp2010-2011/docs/Business_Plan_2010-2011_ENG.pdf].

3. Schoen C, Osborn R, Doty MM, Squires D, Peugh J, Applebaum S: A survey of primary care physicians in eleven countries, 2009: Perspectives on care, costs and experiences. Health Aff 2009, 28(6):21171-21183.

4. Lai JK, Lau F, Shaw N: A study of information technology use and implementation of electronic medical record systems in BC medical practices. BC Medical Journal 2009, 51(3):114-121.

5. Terry AL, Thorpe CF, Giles G, Brown JB, Harris SB, Reid GJ, Thind A, Stewart M: Implementing electronic health records: Key factors in primary care. Can Fam Physician 2008, 54(7):730-736.

6. Adaji $A$, Schattner $P$, Jones $K$ : The use of information technology to enhance diabetes management in primary care: a literature review. Inform Prim Care 2008, 16:229-237.

7. Jordan K, Porcheret M, Croft P: Quality of morbidity coding in general practice computerized medical records: a systematic review. Fam Pract 2004, 21:396-412.

8. Thiru K, Hassey A, Sullivan F: Systematic review of scope and quality of electronic patient record data in primary care. BMJ 2003, 326:1070.

9. Bryan C, Boren SA: The use and effectiveness of electronic clinical decision support tools in the ambulatory/primary care setting: a systematic review of the literature. Inform Prim Care 2008, 16:79-91.

10. van der Kam WJ, Moorman PW, Koppejan-Mulder MJ: Effects of electronic communication in general practice. Int J Med Inform Assoc 2000, 60:59-70.

11. Mitchell E, Sullivan F: A descriptive feast but an evaluative famine: systematic review of published articles on primary care computing during 1980-97. BMJ 2001, 322:279-282.

12. Chan WS, Stevenson M, McGlade K: Do general practitioners change how they use the computer during consultations with a significant psychological component? Intl J Med Inform Assoc 2008, 77:534-538.

13. Keshavjee K, Troyan S, Holbrook AM, VanderMolen D: Measuring the success of electronic medical record implementation using electronic and survey data. In AMIA 2001: Proceedings of the AMIA Annual Symposium 2001; Nov 3-7. Edited by: Bakken S. Washington, DC: Hanley 2001:309-312.
14. Simon SR, Kaushal R, Cleary PD, Jenter CA, Volk LA, Poon EG: Correlates of electronic health record adoption in office practices: A statewide survey. J Am Med Inform Assoc 2007, 14:110-117.

15. Lau F, Kuziemsky C, Price M, Gardner J: A review on systematic reviews of health information system studies. J Am Med Inform Assoc 2010, 17:637-645.

16. Mays N, Pope C, Popay J: Systematically reviewing qualitative and quantitative evidence to inform management and policy-making in the health field. J Health Serv Res Policy 2005, 10:(Suppl 1):S6-S20.

17. Lau F, Price M, Keshavjee K: From benefits evaluation to clinical adoption: making sense of health information system success in Canada. Healthc $Q$ 2011, 14(1):39-45.

18. Lau F, Hagens S, Muttitt S: A proposed benefits evaluation framework for health information systems in Canada. Healthc Q 2007, 10(1):112-118.

19. DeLone WH, McLean ER: Information system success: the quest for the dependent variable. ISR 1992, 3:60-95.

20. DeLone WH, McLean ER: The DeLone and McLean model of information system success: a ten year update. J MIS 2003, 10(4):9-30.

21. Garg AX, Adhikari NKJ, McDonald H, Rosas-Arellano MP, Devereaux PJ, Beyene J, et al: Effects of computerized clinical decision support systems on practitioner performance and patient outcomes: a systematic review. JAMA 2005, 293:1223-1238.

22. Balas EA, Austin SM, Mitchell JA, Ewigman BG, Bopp KD, Brown GD: The clinical value of computerized information services. A review of 98 randomized clinical trials. Arch Fam Med 1996, 5:271-278.

23. Baron RJ: Quality improvement with an electronic health record: achievable, but not automatic. Ann Intern Med 2007, 147:549-552.

24. Bassa A, del Val M, Cobos A, Torremade E, Bergonon S, Crespo C, et al: Impact of a clinical decision support system on the management of patients with hypercholesterolemia in the primary healthcare setting. Dis Manage Health Outcomes 2005, 13:65-72.

25. Bolger-Harris $\mathrm{H}$, Schattner $\mathrm{P}$, Saunders M: Using computer based templates for chronic disease management. Aust Fam Physician 2008, 37:285-288.

26. Booth $\mathrm{N}$, Robinson P, Kohannejad J: Identification of high-quality consultation practice in primary care: the effects of computer use on doctor-patient rapport. Inform Prim Care 2004, 12:75-83.

27. Cauldwell M, Beattie C, Cox B, Denby W, Ede-Golightly J, Linton F: The impact of electronic patient records on workflow in general practice. Health Informatics J 2007, 13:155-160.

28. Christensen T, Grimsmo A: Instant availability of patient records, but dimished availability of patient information: a multi-method study of GP's use of electronic patient records. BMC Med Inform Decis Mak [Internet] 2008, 8(1):12[http://www.biomedcentral.com/1472-6947/8/12].

29. Christensen T, Grimsmo A: Expectations for the next generation of electronic patient records in primary care: a triangulated study. Inform Prim Care 2008, 16:21-28.

30. Crosson JC, Stroebel C, Scott JG, Stello B, Crabtree BF: Implementing an electronic medical record in a family medicine practice: communication, decision making, and conflict. Ann Fam Med 2005, 3:307-311.

31. Crosson JC, Ohman-Strickland PA, Hahn KA, DiCicco-Bloom B, Shaw E, Orzano AJ, et al: Electronic medical records and diabetes quality of care: results from a sample of family medicine practices. Ann Fam Med 2007, 5:209-215.

32. de Jong JD, Groenewegen PP, Spreeuwenberg P, Westert GP, Bakker DH: Do decision support systems influence variation in prescription? BMC Health Serv Res [Internet] 2009, 9(20):15[http://www.biomedcentral.com/ 1472-6963/9/20].

33. Dennison J, Eisen S, Towers M, Ingham Clark C: An effective electronic surgical referral system. Ann Roy Coll Surg Engl 2006, 88:554-556.

34. Filippi A, Sabatini A, Badioli L, Samani F, Mazzaglia G, Catapano A, et al: Effects of an automated electronic reminder in changing the antiplatelet drug-prescribing behavior among italian general practitioners in diabetic patients. Diabetes Care 2003, 26:1497-1500.

35. Frank O, Litt J, Beilby J: Opportunistic electronic reminders: improving performance of preventive care in general practice. Aust Fam Physician 2004, 33:87-90.

36. Hamilton WT, Round AP, Sharp D, Peters TJ: The quality of record keeping in primary care: a comparison of computerised, paper and hybrid systems. Br J Gen Pract 2003, 53:929-933. 
37. Hippisley-Cox J, Pringle M, Cater R, Wynn A, Hammersley V, Coupland C, et al: The electronic patient record in primary care-regression or progression? A cross sectional study. BMJ 2003, 326:1439-1443.

38. Hollingworth W, Devine EB, Hansen RN, Lawless NM, Comstock BA, WilsonNorton $J$, et al: The impact of e-prescribing on prescriber and staff time in ambulatory care clinics: a time-motion study. J Am Med Inform Assoc 2007, 14:722-730.

39. Kenealy T, Arroll B, Petrie KJ: Patients and computers as reminders to screen for diabetes in family practice. Randomized-controlled trial. J Gen Intern Med 2005, 20:916-921.

40. Kinn JW, OToole MF, Rowley SM, Marek JC, Bufalino VJ, Brown AS: Effectiveness of the electronic medical record in cholesterol management in patients with coronary artery disease (Virtual Lipid Clinic). Am J Cardiol 2001, 88:163-165.

41. Linder JA, Ma J, Bates DW, Middleton B, Stafford RS: Electronic health record use and the quality of ambulatory care in the United States. Arch Intern Med 2007, 167:1400-1405.

42. Ludwick DA, Doucette J: Primary care physicians' experience with electronic medical records: barriers to implementation in a fee for service environment. Int J Telemed App/ 2009 [http://www.hindawi.com/ journals/ijta/2009/853524/].

43. Margalit RS, Roter D, Dunevant MA, Larson S, Reis S: Electronic medical record use and physician-patient communication: an observational study of Israeli primary care encounters. Patient Educ Couns 2006, 61:134-141.

44. Martens JD, van der Weijden T, Severens JL, de Clerq PA, de Bruijn DP, Kester ADM, et al: The effect of computer reminders on GPs' prescribing behaviour: a cluster randomised trial. Int J Med Inform 2007, 76(Suppl 3): S403-S416.

45. Miller RH, West C, Brown TM, Sim I, Ganchoff C: The value of electronic health records in solo or small group practices. Health Aff 2005, 24:1127-1137.

46. Miller RH, Sim I, Newman J: Electronic medical records in solo/small groups: a qualitative study of physician user types. In MEDINFO 2004: Proceedings of the 11th World Congress on Medical Informatics; 2004 Sep 711. Edited by: Fieschi M, Coiera E, Li YJ. San Fransico, USA: Ios Press; 2004:658-662.

47. Mitchell E, Sullivan F, Watt G, Grimshaw JM, Donnan PT: Using electronic patient records to inform strategic decision making in primary care. In MEDINFO 2004: Proceedings of the 11th World Congress on Medical Informatics; 2004 Sep 7-11. Edited by: Fieschi M, Coiera E, Li YJ. San Fransico, USA: Ios Press; 2004:1157-1160.

48. Mitchell E, McConnahie A, Sullivan F: Consultation computer use to improve management of chronic disease in general practice: a before and after study. Inform Prim Care 2003, 11:61-68.

49. Montgomery AA, Fahey T, Peters TJ, Maclntosh C, Sharp DJ: Evaluation of computer based clinical decision support system and risk chart for management of hypertension in primary care: randomised controlled trial. BMJ 2000, 320:686-690.

50. Newby DA, Fryer JL, Henry DA: Effect of computerised prescribing on use of antibiotics. Med J Aust 2003, 177:210-213.

51. Poley MJ, Edelenbox Kl, Mosseveld M, van Wijk MA, de Bakker DH, van de Lei J, et al: Cost consequences of implementing an electronic cecision support system for ordering laboratory tests in primary care: evidence from a controlled prospective study in the Netherlands. Clin Chem 2007, 53:213-219.

52. Randeree E: Exploring physician adoption of EMRs: a multi-case analysis. J Med Syst 2007, 31:489-496.

53. Robinson A: Information technology creeps into rural general practice. Aust Health Re 2003, 26(1):131-137.

54. Rollman BL, Hanusa BH, Gilbert T, Lowe HJ, Kapoor WN, Schulberg HC: The electronic medical record: a randomized trial of its impact on primary care physicians' initial management of major depression. Arch Intern Med 2001, 161:189-97, Erratum in: Arch Intern Med 2001, 161:705.

55. Samoutis G, Soteriades E, Kounalakis DK, Zachariadou T, Philalithis A, Lionis C: Implementation of an electronic medical record system in previously computer-naive primary care centres: a pilot study from Cyprus. Inform Prim Care 2007, 15:207-216.
56. Schade CP, Sullivan FM, de Lusignan S, Madeley J: e-Prescribing, efficiency, quality: lessons from the computerization of UK family practice. J Am Med Inform Assoc 2006, 13:470-475.

57. Tamblyn R, Huang A, Taylor L, Kawasumi Y, Bartlett G, Grand R, et al: A randomized trial of the effectiveness of on-demand versus computertriggered drug decision support in primary care. J Med Inform Assoc 2008, 15:430-438.

58. Tamblyn R, Huang A, Perreault R, Jacquies A, Roy D, Hanley J, et al: The medical office of the 21st century (MOXXI): effectiveness of computerized decision-making support in reducing inappropriate prescribing in primary care. CMAJ 2003, 169:549-556.

59. Vainiomaki S, Kuusela M, Vainiomaki P, Rautava P: The quality of electronic patient records in Finnish primary healthcare needs to be improved. Scand J Prim Care 2008, 26:117-22.

60. van Wijk MA, van der Lei J, Mosseveld M, Bohnen AM, van Bemmel JH: Assessment of decision support for blood test ordering in primary care: a randomized trial. Ann Intern Med 2001, 134:274-281.

61. van Wyk JT, van Wijk MA, Sturkenboom MC, Mosseveld M, Moorman PW, van der Lei J: Electronic alerts versus on-demand decision support to improve dyslipidemia treatment: a cluster randomized controlled trial. Circulation 2008, 117:371-378.

62. Wager KA, Lee FW, White AW, Ward DM, Ornstein SM: Impact of an electronic medical record system on community-based primary care practices. J Am Board Fam Pract 2000, 13:338-348.

63. Wells S, Furness S, Rafter N, Horn E, Whittaker R, Stewart A, et al: Integrated electronic decision support increases cardiovascular disease risk assessment four fold in routine primary care practice. Eur J Cardiovasc Prev Rehabil 2008, 15:173-178.

64. Eslami S, de Keizer N, Abu-Hanna A: The impact of computerized physician medication order entry in hsopitalized patients - A systematic review. Intl J Med Inform 2008, 77:365-376

65. Van der Maijden MJ, Tange HJ, Hasman TA: Determinants of success of inpatient clinical information systems: a literature review. J Am Med Inform Assoc 2003, 10(3):235-243.

66. Cooper RB, Zmud RW: Information technology implementation research A technological diffusion approach. Manag Sci 1990, 36:123-139.

67. Hsieh JP-A, Zmud RW: Understanding post-adoptive usage behaviors: A two dimensional view. SIGADIT 2006, Available from http://aisel.aisnet.org/ digit2006/3/

\section{Pre-publication history}

The pre-publication history for this paper can be accessed here: http://www.biomedcentral.com/1472-6947/12/10/prepub

doi:10.1186/1472-6947-12-10

Cite this article as: Lau et al.: Impact of electronic medical record on physician practice in office settings: a systematic review. BMC Medical Informatics and Decision Making 2012 12:10

\section{Submit your next manuscript to BioMed Central and take full advantage of:}

- Convenient online submission

- Thorough peer review

- No space constraints or color figure charges

- Immediate publication on acceptance

- Inclusion in PubMed, CAS, Scopus and Google Scholar

- Research which is freely available for redistribution

Submit your manuscript at www.biomedcentral.com/submit
C Biomed Central 